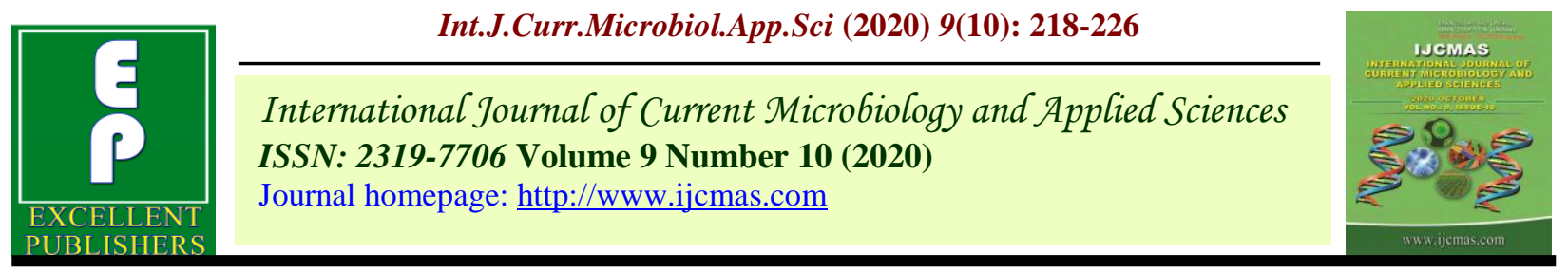

Original Research Article

https://doi.org/10.20546/ijcmas.2020.910.028

\title{
Evaluation of Non-insecticidal Approaches against Pod Borer, Helicoverpa armigera Hubner on Chickpea at S.K. CARS, Kawardha Chhattisgarh
}

\author{
Jai Kishan Bhagat ${ }^{1 *}$, V. K. Soni ${ }^{2}$ and H. K. Chandrakar ${ }^{1}$ \\ ${ }^{1}$ Department of Entomology, College of Agriculture, IGKV, Raipur - 492012 \\ (Chhattisgarh), India \\ ${ }^{2}$ College of Agriculture, Marra, Durg- 491221 (Chhattisgarh, India \\ *Corresponding author
}

\section{A B S T R A C T}

\begin{tabular}{|l|}
\hline Ke y w o r d s \\
$\begin{array}{l}\text { Chickpea, pod } \\
\text { borer, NSKE, (Btk), } \\
\text { Beauveria bassiana, } \\
\text { Karanj oil }\end{array}$ \\
\hline Article Info \\
\hline $\begin{array}{l}\text { Accepted: } \\
\text { 04 September } 2020 \\
\text { Available Online: } \\
\text { 10 October } 2020\end{array}$ \\
\hline
\end{tabular}

\section{Introduction}

Chickpea (Cicer arietinum L.) is the most important pulse crop grown in India.' It is commonly recognized by the name 'Chickpea' or Bengal gram or Chana and belongs to leguminoseae family. It is probably originated from South Eastern Turkey and from there it spread to other countries of the world therefore it is known as 'King of Pulses' (Khatoon and Prakash, 2004). In India, Chickpea crop is being cultivated in an area of about 9626.16 thousand hectares with a production of 9377.56 thousand million tonnes and productivity of $974 \mathrm{~kg} / \mathrm{ha}$.
The present investigation "Evaluation of non-insecticidal approaches against pod borer, Helicoverpa armigera Hubner on chickpea at S.K. CARS, Kawardha Chhattisgarh" was conducted during rabi 2016-17 and 2017-18 at Sant Kabir College of Agriculture \& Research Station, Kawardha Chhattisgarh. The results revealed with mean pooled data that the mean number of larvae per meter row length was low in NSKE treatment (0.78 larva/mrl) Bacillus thuringiensis kurstaki (Btk) (0.92 larva/mrl) and followed by Beauveria bassiana (1.05 larva/mrl) over untreated control (1.74). Similarly, after I $^{\text {st }}$ and II $^{\text {nd }}$ spray the Karanj oil treatment showed 1.16 larva/mrl of $H$. armigera per meter row length.

Chickpea covers an area of about 307.40 thousand hectare with a production of 359.90 thousand million tonnes and productivity of $1171 \mathrm{~kg} / \mathrm{ha}$ in Chhattisgarh (Anonymous, 2017). Chickpea is cultivated as one of the major pulse crops in Kabirdham district of Chhattisgarh acreage 84,100 hectare with productivity of $1040 \mathrm{~kg}$ /ha (Anonymous,2016). Gram pod borer $H$. armigera (Hubner) (Lepidoptera: Noctuidae) is consider as a major pest of Chickpea. As this insect pest is a major hindrance and becomes a universal concern for the production of chickpea (Wubneh, 2016), which cause high economic losses to the 
chickpea crop (Singh and Yadav, 2009). This pest starts infesting the shoot/tips few weeks after crop emergence and feed on buds, flowers and pods till harvesting, causing heavy yield losses. Larvae of $H$. armigera (Hubner) are prodigious foliar feeder as early instars and later move to the developing seeds and fruits leading to drastic reduction in yield. A single larva can consume up to $30-40$ pods in its life cycle (Taggar and Singh, 2012). The plant product like NSKE and other neem derivatives, biopesticides made from neem are non-toxic, biodegradable, ecofriendly and have no residual effect can be used along with
Beauveria bassiana and Bt which has no harmful effect on its most potent parasitoid Campoletis chloridae (Uchida) and effectual in restricting the development of larval population of Helicoverpa armigera on crop.

\section{Materials and Methods}

Management practices against chickpea pod borer, Helicoverpa armigera (Hubner) were studied locations viz., experimental area of $\mathrm{S}$. K. College of Agriculture \& Research Station Kawardha (Kabirdham), Chhattisgarh.

\section{Experiment details}

\begin{tabular}{|l|l|}
\hline Season & $\boldsymbol{R a b i} \mathbf{( 2 0 1 6 - 1 7}$ and 2017-18) \\
\hline Variety & JAKI -9218 \\
\hline Design & RBD \\
\hline No. of Treatments & 05 \\
\hline Replications & 04 \\
\hline Plant spacing & {$[30(\mathrm{~cm})(\mathrm{R} X \mathrm{X})] \mathrm{X}[15(\mathrm{~cm})(\mathrm{P} \mathrm{X} \mathrm{P)}]$} \\
\hline Date of sowing & First week of December \\
\hline Plot size & 20 X 4.5m ${ }^{2}$ \\
\hline Fertilizer application & N.P.K. @ 20:50:20 Kg/ha. \\
\hline
\end{tabular}

\section{Treatment details}

\begin{tabular}{|l|l|l|}
\hline & Treatments & Dose /ha (g or ml) \\
\hline $\mathbf{T}_{\mathbf{1}}$ & NSKE & $5 \%$ \\
\hline $\mathbf{T}_{\mathbf{2}}$ & Karanj Oil & $2 \%$ \\
\hline $\mathbf{T}_{\mathbf{3}}$ & $\begin{array}{l}\text { Bacillus thuringiensis kurstaki } \\
\text { (Bt) }\end{array}$ & $1 \mathrm{~kg} / \mathrm{ha}$ \\
\hline $\mathbf{T}_{\mathbf{4}}$ & Beauveria bassiana & $2.5 \mathrm{Kg} / \mathrm{ha}$ \\
\hline $\mathbf{T}_{\mathbf{5}}$ & Untreated control (plain water) & - \\
\hline
\end{tabular}

In this experiment number of caterpillars of pod borers were counted from randomly selected five different spots of one meter row length area from each plot, pre-treatment on larval population were recorded 1 day before treatment and the post treatment counts were taken after 1, 3, 5, 7, 10 and 15 days after treatment. At the time of harvest five plants from each plot was randomly selected and percent pod damage and grain damage were recorded, by using following formula-

Pod damage $(\%)=\frac{\text { Total no. of damaged pod }}{\text { Total no. of pod }} \times 100$

Yield was calculated under different treatments as given below: 
Yield $/$ ha $=$ Factor $\times$ grain yield $/$ plot

Where,

$$
\text { Factor }=\left(\frac{10000}{\text { Net plotsize }}\right) \text { in sq. m. }
$$

\section{Results and Discussion}

To evaluate the non-insecticidal approaches against pod borer Helicoverpa armigera (Hubner) on chickpea an experiment with 4 botanical insecticides viz., NSKE 5\%, Karanj oil 2\%, Bacillus thuringiensis kurstaki (Btk) 1 $\mathrm{kg} / \mathrm{ha}$ and Beauveria bassiana $2.5 \mathrm{~kg} / \mathrm{ha}$ was laid out in randomized block design (RBD) with four replication along with an untreated check at S. K. College of Agriculture and Research Station, Kawardha during rabi 2016-17 and 2017-18.

Non-insecticidal approaches against pod borer, $\boldsymbol{H}$. armigera Hubner on chickpea during rabi 2016-17

\section{Before spray}

The results presented in Table 1 revealed that the mean number of larvae per meter row length in all the treatments 24 hrs. prior to application of treatments was non-significant or similar.

\section{First spray}

The various treatments with Helicoverpa armigera population ranging from 0.87 to $2.57 \mathrm{larva} / \mathrm{mrl}$ after $15 \mathrm{DAS}$ (Table 1).

Among the different treatments, NSKE 5\% (0.87 larva/mrl) was observed less and at par with Bacillus thuringiensis kurstaki (Btk) 1 $\mathrm{kg} / \mathrm{ha}(0.93 \mathrm{larva} / \mathrm{mrl})$ followed by Beauveria bassiana $2.5 \mathrm{~kg} / \mathrm{ha}$ (1.07 larva/mrl), Karanj oil 2\% (1.27 larva/mrl) and untreated control (2.57 larva/mrl).
The result revealed that 15 days after first spray the NSKE was the most effective at par with $B t$ followed by Beauveria bassiana and Karanj oil was found to be the least effective non-insecticide.

\section{Second spray}

Data presented in Table 1 showed that the effects of all the treatments were similar to the effects found in the first spray.

After second spray at 15 days after application maximum control was observed in NSKE (0.60 larva/mrl) at par with Bacillus thuringiensis kurstaki (Btk) $1 \mathrm{~kg} / \mathrm{ha}(0.63$ larvae/mrl) followed by Beauveria bassiana $2.5 \mathrm{~kg} / \mathrm{ha}$ (0.80 larva/mrl), Karanj oil 2\% (0.87 larva/mrl) and untreated control (0.93 larva/mrl).

\section{Overall mean}

The overall mean showed that mean number of larvae per meter row length was low in NSKE treatment (0.73 larva) at par with $B t$ (0.87 larva/mrl) followed by Beauveria bassiana (1.11 larva/mrl) over untreated control (2.36 larva/mrl).

Similarly, after $\mathrm{I}^{\mathrm{st}}$ and $\mathrm{II}^{\mathrm{nd}}$ spray the Karanj oil treatment showed 1.23 larvae of H.armigera per meter row length.

Non-insecticidal approaches against pod borer, $H$. armigera Hubner on chickpea during rabi 2017-18

\section{Before spray}

The results presented in Table 2 revealed that the mean number of larvae per meter row length in all the treatments $24 \mathrm{hrs}$. prior to application of treatments was non-significant or similar. 
Table.1 Evaluation of non-insecticidal approaches against pod borer, H. armigera (Hub.) on chickpea at S. K. CARS, Kawardha during Rabi 2016-17

\begin{tabular}{|c|c|c|c|c|c|c|c|c|c|c|c|c|c|c|c|c|}
\hline & \multirow[t]{3}{*}{ Treatments } & \multirow[t]{3}{*}{ Dose } & \multicolumn{13}{|c|}{ Mean number of larvae per meter row length } & \multirow{3}{*}{$\begin{array}{c}\text { Overall } \\
\text { mean }\end{array}$} \\
\hline & & & \multirow{2}{*}{$\begin{array}{c}\text { Pre } \\
\text { treatment }\end{array}$} & \multicolumn{6}{|c|}{$1^{\text {st }}$ Spray } & \multicolumn{6}{|c|}{$2^{\text {nd }}$ Spray } & \\
\hline & & & & $\begin{array}{c}1 \\
\text { DAS }\end{array}$ & $\begin{array}{c}3 \\
\text { DAS }\end{array}$ & $\begin{array}{c}5 \\
\text { DAS }\end{array}$ & $\begin{array}{c}7 \\
\text { DAS }\end{array}$ & $\begin{array}{c}10 \\
\text { DAS }\end{array}$ & $\begin{array}{c}15 \\
\text { DAS }\end{array}$ & $\begin{array}{c}1 \\
\text { DAS }\end{array}$ & $\begin{array}{c}3 \\
\text { DAS }\end{array}$ & $\begin{array}{c}5 \\
\text { DAS }\end{array}$ & $\begin{array}{c}7 \\
\text { DAS }\end{array}$ & $\begin{array}{c}10 \\
\text { DAS }\end{array}$ & $\begin{array}{c}15 \\
\text { DAS }\end{array}$ & \\
\hline $\mathbf{T}_{1}$ & NSKE & $5 \%$ & $\begin{array}{c}1.60 \\
(1.61)\end{array}$ & $\begin{array}{c}0.87 \\
(1.36)\end{array}$ & $\begin{array}{c}0.67 \\
(1.29)\end{array}$ & $\begin{array}{c}0.47 \\
(1.21)\end{array}$ & $\begin{array}{c}0.73 \\
(1.31)\end{array}$ & $\begin{array}{c}0.93 \\
(1.38)\end{array}$ & $\begin{array}{c}0.87 \\
(1.36)\end{array}$ & $\begin{array}{c}1.00 \\
(1.41)\end{array}$ & $\begin{array}{c}0.53 \\
(1.23)\end{array}$ & $\begin{array}{c}1.33 \\
(1.52)\end{array}$ & $\begin{array}{c}0.40 \\
(1.18)\end{array}$ & $\begin{array}{c}0.33 \\
(1.15)\end{array}$ & $\begin{array}{c}0.60 \\
(1.26)\end{array}$ & $\begin{array}{c}0.73 \\
(1.31)\end{array}$ \\
\hline $\mathbf{T}_{2}$ & Karanj Oil & $2 \%$ & $\begin{array}{l}1.20 \\
(1.48)\end{array}$ & $\begin{array}{c}1.20 \\
(1.48)\end{array}$ & $\begin{array}{c}1.13 \\
(1.46)\end{array}$ & $\begin{array}{c}0.86 \\
(1.35)\end{array}$ & $\begin{array}{c}1.20 \\
(1.48)\end{array}$ & $\begin{array}{l}1.33 \\
(1.52)\end{array}$ & $\begin{array}{c}1.27 \\
(1.51)\end{array}$ & $\begin{array}{c}1.53 \\
(1.59)\end{array}$ & $\begin{array}{l}1.80 \\
(1.67)\end{array}$ & $\begin{array}{c}1.73 \\
(1.65)\end{array}$ & $\begin{array}{c}0.93 \\
(1.39)\end{array}$ & $\begin{array}{c}0.81 \\
(1.33)\end{array}$ & $\begin{array}{c}0.87 \\
(1.36)\end{array}$ & $\begin{array}{c}1.23 \\
(1.50)\end{array}$ \\
\hline $\mathbf{T}_{3}$ & $\begin{array}{l}\text { Bacillus thuringiensis } \\
\text { kurstaki (Btk) }\end{array}$ & $\begin{array}{c}1 \\
\mathrm{~kg} / \mathrm{ha}\end{array}$ & $\begin{array}{c}1.60 \\
(1.61)\end{array}$ & $\begin{array}{c}0.83 \\
(1.32)\end{array}$ & $\begin{array}{c}0.67 \\
(1.30)\end{array}$ & $\begin{array}{c}0.69 \\
(1.30)\end{array}$ & $\begin{array}{c}0.87 \\
(1.36)\end{array}$ & $\begin{array}{c}1.07 \\
(1.43)\end{array}$ & $\begin{array}{c}0.93 \\
(1.39)\end{array}$ & $\begin{array}{c}1.13 \\
(1.46)\end{array}$ & $\begin{array}{c}0.80 \\
(1.34)\end{array}$ & $\begin{array}{c}1.47 \\
(1.56)\end{array}$ & $\begin{array}{c}0.73 \\
(1.31)\end{array}$ & $\begin{array}{c}0.60 \\
(1.26)\end{array}$ & $\begin{array}{c}0.63 \\
(1.27)\end{array}$ & $\begin{array}{c}0.87 \\
(1.36)\end{array}$ \\
\hline $\mathbf{T}_{4}$ & Beauveria bassiana & $\begin{array}{c}2.5 \\
\mathrm{~kg} / \mathrm{ha}\end{array}$ & $\begin{array}{l}1.67 \\
(1.63)\end{array}$ & $\begin{array}{c}0.93 \\
(1.39)\end{array}$ & $\begin{array}{c}0.73 \\
(1.31)\end{array}$ & $\begin{array}{c}0.67 \\
(1.29)\end{array}$ & $\begin{array}{c}1.00 \\
(1.41)\end{array}$ & $\begin{array}{l}1.47 \\
(1.57)\end{array}$ & $\begin{array}{l}1.07 \\
(1.44)\end{array}$ & $\begin{array}{c}1.13 \\
(1.46)\end{array}$ & $\begin{array}{c}1.04 \\
(1.42)\end{array}$ & $\begin{array}{c}1.67 \\
(1.63)\end{array}$ & $\begin{array}{c}1.20 \\
(1.48)\end{array}$ & $\begin{array}{c}1.60 \\
(1.61)\end{array}$ & $\begin{array}{c}0.80 \\
(1.34)\end{array}$ & $\begin{array}{c}1.11 \\
(1.42)\end{array}$ \\
\hline $\mathbf{T}_{5}$ & $\begin{array}{l}\text { Untreated control (plain } \\
\text { water) }\end{array}$ & & $\begin{array}{c}1.87 \\
(1.69)\end{array}$ & $\begin{array}{c}2.13 \\
(1.77)\end{array}$ & $\begin{array}{c}2.33 \\
(1.82)\end{array}$ & $\begin{array}{c}2.37 \\
(1.84)\end{array}$ & $\begin{array}{c}2.43 \\
(1.87)\end{array}$ & $\begin{array}{c}2.03 \\
(1.76)\end{array}$ & $\begin{array}{c}2.57 \\
(1.77)\end{array}$ & $\begin{array}{c}2.27 \\
(1.80)\end{array}$ & $\begin{array}{c}2.53 \\
(1.88)\end{array}$ & $\begin{array}{c}2.47 \\
(1.86)\end{array}$ & $\begin{array}{c}2.40 \\
(1.84)\end{array}$ & $\begin{array}{c}2.46 \\
(1.86)\end{array}$ & $\begin{array}{c}2.33 \\
(1.83)\end{array}$ & $\begin{array}{c}2.36 \\
(1.85)\end{array}$ \\
\hline & SE $(\mathbf{m}) \pm$ & & 0.06 & 0.03 & 0.03 & 0.02 & 0.04 & 0.03 & 0.04 & 0.04 & 0.03 & 0.02 & 0.02 & 0.03 & 0.02 & 0.05 \\
\hline & CD $5 \%$ & & NS & 0.09 & 0.09 & 0.07 & 0.12 & 0.09 & 0.13 & 0.11 & 0.10 & 0.09 & 0.08 & 0.09 & 0.08 & 0.15 \\
\hline
\end{tabular}

Note: Figures in parentheses are Square root transformed value, DAS= Days after spray

Table.2 Evaluation of non-insecticidal approaches against pod borer, H. armigera (Hub.) on chickpea at S. K. CARS, Kawardha during Rabi 2017-18

\begin{tabular}{|c|c|c|c|c|c|c|c|c|c|c|c|c|c|c|c|c|}
\hline & \multirow[t]{3}{*}{ Treatments } & \multirow[t]{3}{*}{ Dose } & \multicolumn{13}{|c|}{ Mean number of larvae per meter row length } & \multirow{3}{*}{$\begin{array}{c}\text { Overal } \\
\text { mean }\end{array}$} \\
\hline & & & \multirow{2}{*}{$\begin{array}{c}\text { Pre } \\
\text { treatment }\end{array}$} & \multicolumn{6}{|c|}{$1^{\text {st }}$ Spray } & \multicolumn{6}{|c|}{$2^{\text {nd }}$ Spray } & \\
\hline & & & & $\begin{array}{c}1 \\
\text { DAS }\end{array}$ & 3 DAS & 5 DAS & 7 DAS & $\begin{array}{c}10 \\
\text { DAS }\end{array}$ & $\begin{array}{c}15 \\
\text { DAS }\end{array}$ & $\begin{array}{c}1 \\
\text { DAS }\end{array}$ & 3 DAS & 5 DAS & 7 DAS & $\begin{array}{c}10 \\
\text { DAS }\end{array}$ & $\begin{array}{c}15 \\
\text { DAS }\end{array}$ & \\
\hline $\mathbf{T}_{1}$ & NSKE & $5 \%$ & $\begin{array}{c}1.67 \\
(1.63)\end{array}$ & $\begin{array}{c}1.33 \\
(1.53)\end{array}$ & $\begin{array}{c}1.27 \\
(1.51)\end{array}$ & $\begin{array}{c}0.80 \\
(1.51)\end{array}$ & $\begin{array}{c}0.67 \\
(1.29)\end{array}$ & $\begin{array}{c}0.87 \\
(1.37)\end{array}$ & $\begin{array}{c}0.82 \\
(1.44)\end{array}$ & $\begin{array}{c}1.20 \\
(1.48)\end{array}$ & $\begin{array}{c}0.80 \\
(1.34)\end{array}$ & $\begin{array}{c}0.33 \\
(1.15)\end{array}$ & $\begin{array}{c}0.27 \\
(1.13)\end{array}$ & $\begin{array}{c}0.53 \\
(1.24)\end{array}$ & $\begin{array}{c}0.80 \\
(1.34)\end{array}$ & $\begin{array}{c}\mathbf{0 . 8 3} \\
(\mathbf{1 . 3 6})\end{array}$ \\
\hline $\mathbf{T}_{\mathbf{2}}$ & Karanj Oil & $2 \%$ & $\begin{array}{c}1.60 \\
(1.61)\end{array}$ & $\begin{array}{c}1.27 \\
(1.51)\end{array}$ & $\begin{array}{c}1.30 \\
(1.53)\end{array}$ & $\begin{array}{c}0.60 \\
(1.50)\end{array}$ & $\begin{array}{c}1.07 \\
(1.44)\end{array}$ & $\begin{array}{c}1.25 \\
(1.49)\end{array}$ & $\begin{array}{c}1.13 \\
(1.45)\end{array}$ & $\begin{array}{c}1.00 \\
(1.41)\end{array}$ & $\begin{array}{c}1.12 \\
(1.44)\end{array}$ & $\begin{array}{c}0.60 \\
(1.27)\end{array}$ & $\begin{array}{c}0.80 \\
(1.34)\end{array}$ & $\begin{array}{c}1.27 \\
(1.50)\end{array}$ & $\begin{array}{c}1.26 \\
(1.61)\end{array}$ & $\begin{array}{c}1.09 \\
(1.43)\end{array}$ \\
\hline $\mathbf{T}_{3}$ & $\begin{array}{c}\text { Bacillus thuringiensis } \\
\text { kurstaki (Btk) }\end{array}$ & $1 \mathrm{~kg} / \mathrm{ha}$ & $\begin{array}{c}1.87 \\
(1.69)\end{array}$ & $\begin{array}{c}1.54 \\
(1.63)\end{array}$ & $\begin{array}{c}1.33 \\
(1.53)\end{array}$ & $\begin{array}{c}1.00 \\
(1.41)\end{array}$ & $\begin{array}{c}0.83 \\
(1.32)\end{array}$ & $\begin{array}{c}1.00 \\
(1.41)\end{array}$ & $\begin{array}{c}0.87 \\
(1.37)\end{array}$ & $\begin{array}{c}1.27 \\
(1.51)\end{array}$ & $\begin{array}{c}0.73 \\
(1.32)\end{array}$ & $\begin{array}{c}0.53 \\
(1.24)\end{array}$ & $\begin{array}{c}0.33 \\
(1.15)\end{array}$ & $\begin{array}{c}0.72 \\
(1.32)\end{array}$ & $\begin{array}{c}0.93 \\
(1.39)\end{array}$ & $\begin{array}{c}0.96 \\
(1.40)\end{array}$ \\
\hline $\mathbf{T}_{4}$ & Beauveria bassiana & $2.5 \mathrm{~kg} / \mathrm{ha}$ & $\begin{array}{c}1.73 \\
(1.65)\end{array}$ & $\begin{array}{c}1.53 \\
(1.59)\end{array}$ & $\begin{array}{c}1.27 \\
(1.51)\end{array}$ & $\begin{array}{c}0.87 \\
(1.37)\end{array}$ & $\begin{array}{c}0.80 \\
(1.34)\end{array}$ & $\begin{array}{c}0.93 \\
(1.39)\end{array}$ & $\begin{array}{c}1.10 \\
(1.44)\end{array}$ & $\begin{array}{c}1.12 \\
(1.46)\end{array}$ & $\begin{array}{c}0.87 \\
(1.37)\end{array}$ & $\begin{array}{c}0.93 \\
(1.39)\end{array}$ & $\begin{array}{c}0.73 \\
(1.32)\end{array}$ & $\begin{array}{c}0.93 \\
(1.39)\end{array}$ & $\begin{array}{c}1.07 \\
(1.44)\end{array}$ & $\begin{array}{c}0.99 \\
(1.41)\end{array}$ \\
\hline $\mathbf{T}_{5}$ & $\begin{array}{l}\text { Untreated control (plain } \\
\text { water) }\end{array}$ & & $\begin{array}{c}2.07 \\
(1.75)\end{array}$ & $\begin{array}{c}1.67 \\
(1.63)\end{array}$ & $\begin{array}{c}1.40 \\
(1.54)\end{array}$ & $\begin{array}{c}1.13 \\
(1.45)\end{array}$ & $\begin{array}{c}0.73 \\
(1.32)\end{array}$ & $\begin{array}{c}0.73 \\
(1.31)\end{array}$ & $\begin{array}{c}1.28 \\
(1.46)\end{array}$ & $\begin{array}{c}1.37 \\
(1.58)\end{array}$ & $\begin{array}{c}1.20 \\
(1.48)\end{array}$ & $\begin{array}{c}1.13 \\
(1.46)\end{array}$ & $\begin{array}{c}0.87 \\
(1.37)\end{array}$ & $\begin{array}{c}1.10 \\
(1.44)\end{array}$ & $\begin{array}{c}1.32 \\
(1.54)\end{array}$ & $\begin{array}{c}1.12 \\
(1.46)\end{array}$ \\
\hline & SE $(m) \pm$ & & 0.08 & 0.05 & 0.05 & 0.05 & 0.06 & 0.04 & 0.07 & 0.04 & 0.04 & $\mathbf{0 . 0 3}$ & 0.04 & 0.04 & 0.05 & 0.049 \\
\hline & CD 5\% & & NS & 0.18 & 0.15 & 0.15 & 0.17 & 0.12 & 0.21 & 0.12 & 0.13 & 0.10 & 0.13 & 0.12 & 0.15 & 0.14 \\
\hline
\end{tabular}

Note: Figures in parentheses are Square root transformed value, DAS= Days after spray 
Table.3 Evaluation of non-insecticidal approaches against pod borer, H. armigera (Hub.) on chickpea at S. K. CARS, Kawardha (mean pooled data)

\begin{tabular}{|c|c|c|c|c|c|c|c|c|c|c|c|c|c|c|c|c|}
\hline & \multirow[t]{3}{*}{ Treatments } & \multirow[t]{3}{*}{ Dose } & \multicolumn{13}{|c|}{ Mean number of larvae per meter row length } & \multirow{3}{*}{$\begin{array}{c}\text { Overall } \\
\text { mean }\end{array}$} \\
\hline & & & \multirow{2}{*}{$\begin{array}{c}\text { Pre } \\
\text { treatment }\end{array}$} & \multicolumn{6}{|c|}{$1^{\text {st }}$ Spray } & \multicolumn{6}{|c|}{$2^{\text {nd }}$ Spray } & \\
\hline & & & & $\begin{array}{c}1 \\
\text { DAS }\end{array}$ & 3 DAS & 5 DAS & 7 DAS & $\begin{array}{c}10 \\
\text { DAS }\end{array}$ & $\begin{array}{c}15 \\
\text { DAS }\end{array}$ & $\begin{array}{c}1 \\
\text { DAS }\end{array}$ & 3 DAS & 5 DAS & 7 DAS & $\begin{array}{c}10 \\
\text { DAS }\end{array}$ & $\begin{array}{c}15 \\
\text { DAS }\end{array}$ & \\
\hline $\mathbf{T}_{1}$ & NSKE & $5 \%$ & $\begin{array}{c}1.64 \\
(1.62)\end{array}$ & $\begin{array}{c}1.10 \\
(1.45)\end{array}$ & $\begin{array}{c}0.97 \\
(1.40)\end{array}$ & $\begin{array}{c}0.64 \\
(1.27)\end{array}$ & $\begin{array}{c}0.70 \\
(1.30)\end{array}$ & $\begin{array}{c}0.90 \\
(1.37)\end{array}$ & $\begin{array}{c}0.97 \\
(1.40)\end{array}$ & $\begin{array}{c}1.10 \\
(1.45)\end{array}$ & $\begin{array}{c}0.67 \\
(1.29)\end{array}$ & $\begin{array}{c}0.83 \\
(1.35)\end{array}$ & $\begin{array}{c}0.34 \\
(1.15)\end{array}$ & $\begin{array}{c}0.43 \\
(1.19)\end{array}$ & $\begin{array}{c}0.70 \\
(1.30)\end{array}$ & $\begin{array}{c}\mathbf{0 . 7 8} \\
(\mathbf{1 . 3 3})\end{array}$ \\
\hline $\mathbf{T}_{2}$ & Karanj Oil & $2 \%$ & $\begin{array}{c}1.40 \\
(1.54)\end{array}$ & $\begin{array}{c}1.24 \\
(1.49)\end{array}$ & $\begin{array}{c}1.22 \\
(1.48)\end{array}$ & $\begin{array}{c}0.74 \\
(1.31)\end{array}$ & $\begin{array}{c}1.14 \\
(1.46)\end{array}$ & $\begin{array}{c}1.30 \\
(1.51)\end{array}$ & $\begin{array}{l}1.20 \\
(1.48)\end{array}$ & $\begin{array}{c}1.27 \\
(1.50)\end{array}$ & $\begin{array}{c}1.46 \\
(1.56)\end{array}$ & $\begin{array}{c}1.17 \\
(1.47)\end{array}$ & $\begin{array}{c}0.87 \\
(1.36)\end{array}$ & $\begin{array}{c}1.10 \\
(1.44)\end{array}$ & $\begin{array}{c}1.24 \\
(1.49)\end{array}$ & $\begin{array}{c}1.16 \\
(1.47)\end{array}$ \\
\hline $\mathbf{T}_{3}$ & $\begin{array}{l}\text { Bacillus thuringiensis } \\
\text { kurstaki (Btk) }\end{array}$ & $\begin{array}{c}1 \\
\mathrm{~kg} / \mathrm{ha}\end{array}$ & $\begin{array}{c}1.74 \\
(1.65)\end{array}$ & $\begin{array}{c}1.21 \\
(1.48)\end{array}$ & $\begin{array}{c}1.00 \\
(1.41)\end{array}$ & $\begin{array}{c}0.84 \\
(1.35)\end{array}$ & $\begin{array}{c}0.82 \\
(1.34)\end{array}$ & $\begin{array}{c}1.04 \\
(1.42)\end{array}$ & $\begin{array}{c}1.03 \\
(1.42)\end{array}$ & $\begin{array}{c}1.20 \\
(1.48)\end{array}$ & $\begin{array}{c}0.77 \\
(1.32)\end{array}$ & $\begin{array}{c}1.00 \\
(1.41)\end{array}$ & $\begin{array}{c}0.53 \\
(1.23)\end{array}$ & $\begin{array}{c}0.67 \\
(1.29)\end{array}$ & $\begin{array}{c}0.87 \\
(1.36)\end{array}$ & $\begin{array}{c}0.92 \\
(1.37)\end{array}$ \\
\hline $\mathbf{T}_{4}$ & Beauveria bassiana & $\begin{array}{c}2.5 \\
\mathrm{~kg} / \mathrm{ha}\end{array}$ & $\begin{array}{c}1.70 \\
(1.64)\end{array}$ & $\begin{array}{c}1.23 \\
(1.49)\end{array}$ & $\begin{array}{c}1.13 \\
(1.45)\end{array}$ & $\begin{array}{c}0.74 \\
(1.31)\end{array}$ & $\begin{array}{c}0.90 \\
(1.37)\end{array}$ & $\begin{array}{c}1.20 \\
(1.48\end{array}$ & $\begin{array}{c}1.14 \\
(1.46)\end{array}$ & $\begin{array}{c}1.13 \\
(1.45)\end{array}$ & $\begin{array}{c}0.97 \\
(1.40)\end{array}$ & $\begin{array}{c}1.30 \\
(1.51)\end{array}$ & $\begin{array}{c}0.97 \\
(1.40)\end{array}$ & $\begin{array}{c}1.27 \\
(1.50)\end{array}$ & $\begin{array}{c}0.94 \\
(1.39)\end{array}$ & $\begin{array}{c}1.05 \\
(1.42)\end{array}$ \\
\hline \multirow[t]{3}{*}{$\mathbf{T}_{5}$} & $\begin{array}{l}\text { Untreated control (plain } \\
\text { water) }\end{array}$ & & $\begin{array}{c}1.97 \\
(1.72)\end{array}$ & $\begin{array}{c}1.90 \\
(1.70)\end{array}$ & $\begin{array}{c}1.87 \\
(1.69)\end{array}$ & $\begin{array}{l}1.75 \\
(1.65)\end{array}$ & $\begin{array}{c}1.58 \\
(1.60)\end{array}$ & $\begin{array}{c}1.38 \\
(1.54)\end{array}$ & $\begin{array}{l}1.85 \\
(1.68)\end{array}$ & $\begin{array}{c}1.82 \\
(1.67)\end{array}$ & $\begin{array}{c}1.87 \\
(1.69)\end{array}$ & $\begin{array}{c}1.70 \\
(1.64)\end{array}$ & $\begin{array}{c}1.64 \\
(1.62)\end{array}$ & $\begin{array}{c}1.77 \\
(1.66)\end{array}$ & $\begin{array}{c}1.73 \\
(1.65)\end{array}$ & $\begin{array}{c}1.74 \\
(1.64)\end{array}$ \\
\hline & SE $(\mathbf{m}) \pm$ & & 0.05 & 0.01 & 0.01 & 0.02 & 0.02 & 0.01 & 0.01 & 0.01 & 0.01 & 0.01 & 0.02 & 0.01 & $\mathbf{0 . 0 3}$ & 0.06 \\
\hline & CD 5\% & & NS & $\mathbf{0 . 0 3}$ & 0.03 & 0.08 & 0.09 & $\mathbf{0 . 0 3}$ & 0.03 & 0.04 & $\mathbf{0 . 0 3}$ & $\mathbf{0 . 0 3}$ & 0.08 & $\mathbf{0 . 0 3}$ & 0.09 & 0.18 \\
\hline
\end{tabular}

Note: Figures in parentheses are Square root transformed value, DAS= Days after spray

Table.4 Percentage pod damage by pod borer, Helicoverpa armigera (Hub.) in Chickpea at S. K. CARS, Kawardha

\begin{tabular}{|c|c|c|c|c|c|}
\hline & \multirow[t]{2}{*}{ Treatments } & \multirow[t]{2}{*}{ Dose } & \multicolumn{3}{|c|}{ Percentage pod Damage } \\
\hline & & & 2016 & 2017 & pooled \\
\hline $\mathbf{T}_{1}$ & NSKE & $5 \%$ & $\begin{array}{c}4.13 \\
(11.70)\end{array}$ & $\begin{array}{c}3.81 \\
(11.24)\end{array}$ & $\begin{array}{c}3.97 \\
(11.36)\end{array}$ \\
\hline $\mathbf{T}_{2}$ & Karanj Oil & $2 \%$ & $\begin{array}{c}8.34 \\
(16.77)\end{array}$ & $\begin{array}{c}7.11 \\
(15.45)\end{array}$ & $\begin{array}{c}7.73 \\
(15.64)\end{array}$ \\
\hline $\mathbf{T}_{3}$ & $\begin{array}{l}\text { Bacillus thuringiensis kurstaki } \\
\text { (Btk) }\end{array}$ & $1 \mathrm{~kg} / \mathrm{ha}$ & $\begin{array}{c}4.78 \\
(12.56)\end{array}$ & $\begin{array}{c}4.12 \\
(11.65)\end{array}$ & $\begin{array}{c}4.45 \\
(12.32)\end{array}$ \\
\hline $\mathbf{T}_{4}$ & Beauveria bassiana & $2.5 \mathrm{~kg} / \mathrm{ha}$ & $\begin{array}{c}7.44 \\
(15.81)\end{array}$ & $\begin{array}{c}6.83 \\
(15.13)\end{array}$ & $\begin{array}{c}7.14 \\
(15.63)\end{array}$ \\
\hline $\mathbf{T}_{5}$ & Untreated control (plain water) & & $\begin{array}{c}11.89 \\
(20.16)\end{array}$ & $\begin{array}{c}9.75 \\
(18.14)\end{array}$ & $\begin{array}{c}10.82 \\
(19.43)\end{array}$ \\
\hline \multicolumn{3}{|c|}{$\mathrm{SE}(\mathrm{m}) \pm$} & 0.21 & 0.20 & 0.21 \\
\hline \multicolumn{3}{|c|}{ CD 5\% } & 0.67 & 0.64 & 0.66 \\
\hline
\end{tabular}


Table.5 Yield of chickpea at S. K. CARS, Kawardha

\begin{tabular}{|l|l|c|c|c|}
\hline & \multicolumn{1}{|c|}{ Treatments } & \multicolumn{3}{c|}{ Q/ ha. } \\
\cline { 3 - 5 } & & $\mathbf{2 0 1 6 - 1 7}$ & $\mathbf{2 0 1 7 - 1 8}$ & Pooled \\
\hline $\mathbf{T}_{\mathbf{1}}$ & NSKE & 16.26 & 17.20 & 16.73 \\
\hline $\mathbf{T}_{\mathbf{2}}$ & Karanj Oil & 10.42 & 11.46 & 10.94 \\
\hline $\mathbf{T}_{\mathbf{3}}$ & Bacillus thuringiensis kurstaki $($ Btk) & 14.31 & 15.52 & 14.91 \\
\hline $\mathbf{T}_{\mathbf{4}}$ & Beauveria bassiana & 11.62 & 12.12 & 11.87 \\
\hline $\mathbf{T}_{\mathbf{5}}$ & Untreated control (plain water) & 8.34 & 9.04 & 8.69 \\
\hline & SE (m) \pm & $\mathbf{0 . 7 7}$ & $\mathbf{0 . 8 4}$ & $\mathbf{0 . 8 0}$ \\
\hline & CD 5\% & $\mathbf{2 . 2 4}$ & $\mathbf{2 . 4 4}$ & $\mathbf{2 . 3 4}$ \\
\hline
\end{tabular}

Fig.1 Percentage pod damage by pod borer, Helicoverpa armigera Hubner in Chickpea at S. K. CARS, Kawardha

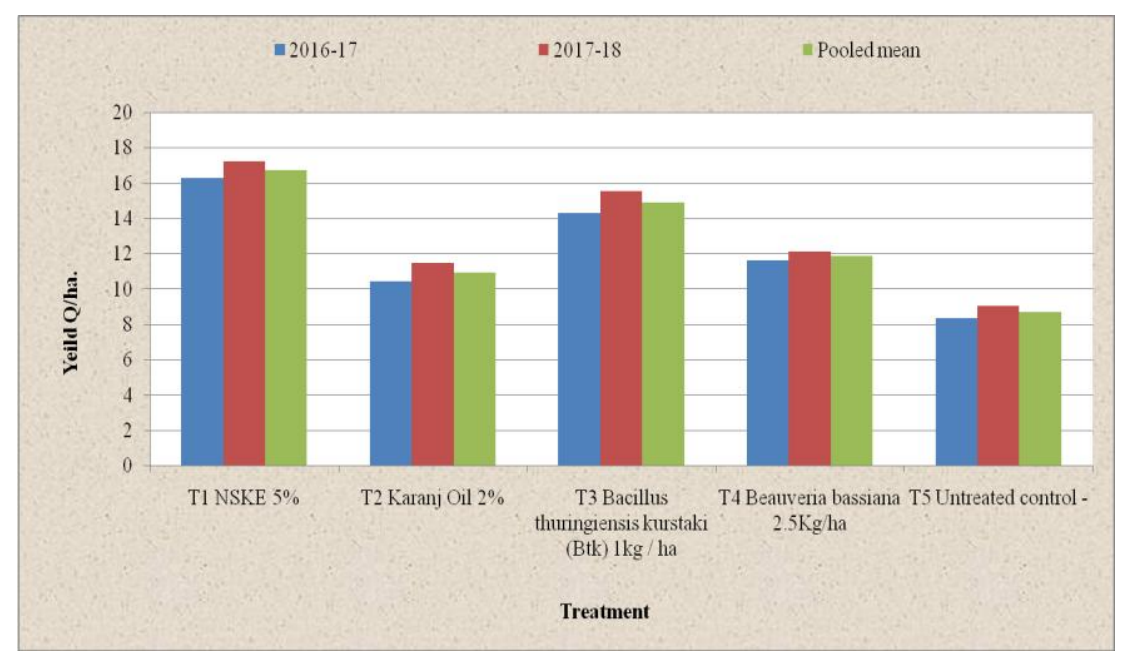

Fig.2 Yield of chickpea at S. K. CARS, Kawardha

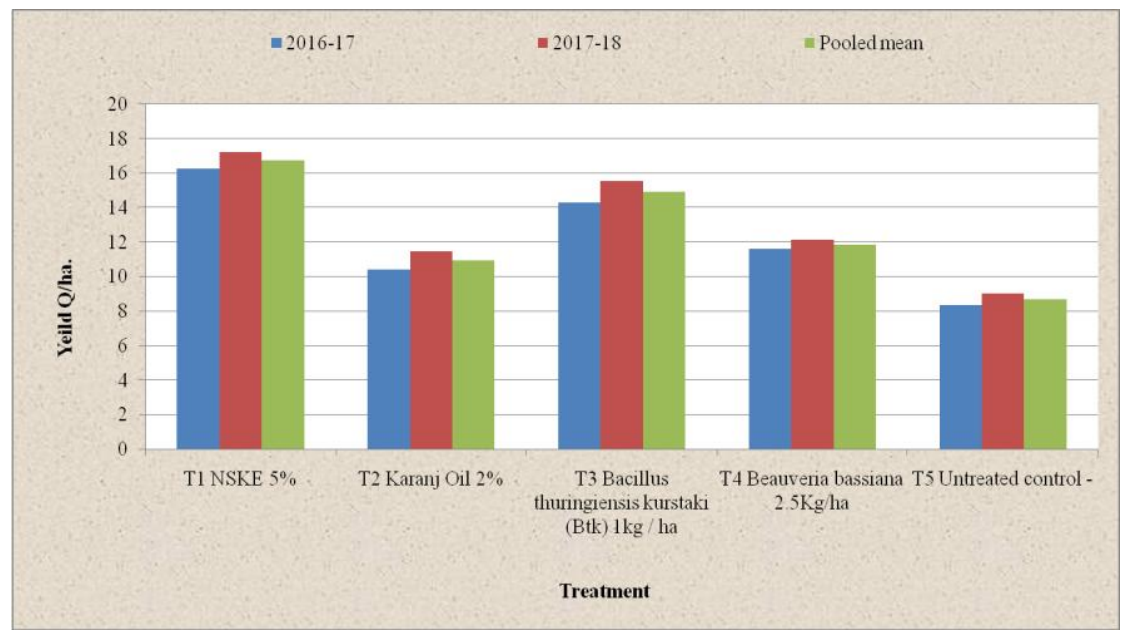




\section{First spray}

The various treatments with Helicoverpa armigera population ranging from 0.82 to 1.28 larva/mrl after 15 DAS (Table 2). Among the different treatments, NSKE 5\% (0.82 larva/mrl) was observed and at par with Bacillus thuringiensis kurstaki (Btk) $1 \mathrm{~kg} / \mathrm{ha}$ (0.87 larva/mrl) followed by Beauveria bassiana $2.5 \mathrm{~kg} / \mathrm{ha}$ (1.10 larvae /mrl), Karanj oil 2\% (1.13 larva/mrl) and untreated control (1.28 larva/mrl).

The result revealed that 15 days after first spray NSKE was the most effective at par with Bacillus thuringiensis kurstaki (Btk) followed by Beauveria bassiana, Karanj oil was found to be the least effective noninsecticide.

\section{Second spray}

Data presented in Table 2 showed that the effects of all the treatments were similar to the effects found in the first spray. After second spray at 15 days after application maximum control was observed in NSKE (0.80 larva/mrl) at par with Bacillus thuringiensis kurstaki (Btk) $1 \mathrm{~kg} / \mathrm{ha}$ (0.93 larva/mrl) followed by Beauveria bassiana $2.5 \mathrm{~kg} / \mathrm{ha}$ (1.07 larva/mrl), Karanj oil 2\% (1.26 larva/mrl) and untreated control (1.32 larva/mrl).

\section{Overall Mean}

The overall mean showed that mean number of larvae per meter row length was low in NSKE treatment (0.83 larva) at par with $B t$ (0.96 larva/mrl) followed by Beauveria bassiana (0.99 larva/mrl) over untreated control (1.12 larva/mrl). Similarly, after $\mathrm{I}^{\text {st }}$ and II $^{\text {nd }}$ spray the Karanj oil treatment showed 1.09 larva of $H$. armigera per meter row length.
Non-insecticidal approaches against pod borer, H.armigera Hubner on chickpea during 2016-17 and 2017-18 (Mean pooled data)

\section{Before spray}

The results presented in Table 3 revealed that the pooled mean of number of larvae per meter row length in all the treatments $24 \mathrm{hrs}$. Prior to application of treatments was nonsignificant or similar.

\section{First spray}

The various treatments with Helicoverpa armigera population ranging from 0.97 to 1.85 larva/mrl after 15 DAS (Table 3). Among the different treatments, NSKE 5\% (0.97 larva/mrl) was observed at par with Bacillus thuringiensis kurstaki (Btk) $1 \mathrm{~kg} / \mathrm{ha}$ (1.03 larvae $/ \mathrm{mrl}$ ) followed by Beauveria bassiana $2.5 \mathrm{~kg} / \mathrm{ha}$ (1.14 larva/mrl), Karanj oil $2 \%$ (1.20 larva/mrl) and untreated control (1.85 larva/mrl).

The result revealed that 15 days after first spray the NSKE was the most effective at par with Bacillus thuringiensis kurstaki (Btk), followed by Beauveria bassiana and Karanj oil was found to be the least effective noninsecticide.

\section{Second Spray}

Data presented in Table 3 showed that the effects of all the treatments were similar to the effects found in the first spray. After second spray at 15 days after application maximum control was observed in NSKE (0.70 larvae/mrl) at par with Bacillus thuringiensis kurstaki (Btk) $1 \mathrm{~kg} / \mathrm{ha}(0.87$ larvae/mrl) followed by Beauveria bassiana $2.5 \mathrm{~kg} / \mathrm{ha}$ (0.94 larvae/mrl), Karanj oil 2\% (1.24 larva/mrl) and untreated control (1.73 larva/mrl) 


\section{Overall Mean}

The overall mean showed that mean number of larvae per meter row length was low in NSKE treatment (0.78 larva/mrl) Bacillus thuringiensis kurstaki (Btk) (0.92 larva/mrl) and followed by Beauveria bassiana (1.05 larva/mrl) over untreated control (1.74). Similarly, after $\mathrm{I}^{\mathrm{st}}$ and $\mathrm{II}^{\mathrm{nd}}$ spray the Karanj oil treatment showed 1.16 larva/mrl of $H$. armigera per meter row length.

The present findings are in accordance with the findings of Anandhi et al., (2011) who studied, neem seed kernel extract (NSKE) treatments was the best treatments with the highest reduction of pod borer population with $40.0 \%$ larval mortality in the first and second spray among the all treatments.

Ahmed et al., (2015) and Zahra et al., (2014) have also concluded that the different treatments of botanicals and microbial had widely varying adverse effects on of $H$. armigera and they observed that treatment of Azadirachtin had higher impact followed by microbial HaNPV, Bacillus thuringiensis kurstaki (Btk) and Beauveria bassiana during both the experimental years.

\section{Pod damage}

The results showed that all the treatments significantly reduced the pod damage due to $H$. armigera (Table 4 and Fig. 1). During 2016 and 2017, there was significantly difference between the treatments with regard to pod damage due to $H$. armigera.

During 2016-17, the pod damage per cent was significantly reduced in treatments with NSKE (4.13\%) at par with Bacillus thuringiensis kurstaki (Btk) (4.78\%) followed by Beauveria bassiana (7.44\%) and Karanj oil $(8.34 \%)$. The untreated control plot recorded pod damage of 11.89 per cent due to
H. armigera during 2017-18, the pod damage per cent was significantly reduced in treatments with NSKE (3.81\%) at par with Bacillus thuringiensis kurstaki (Btk) (4.12\%) followed by Beauveria bassiana (6.83\%) and Karanj oil (7.11\%). The untreated control plot recorded pod damage of 9.75 per cent due to H. armigera.

However on the basis of pooled mean data, the pod damage per cent was significantly reduced in treatments with $\operatorname{NSKE}(3.97 \%)$ at par with Bacillus thuringiensis kurstaki (Btk) (4.45\%) followed by Beauveria bassiana (7.14\%) and Karanj oil (7.73\%). The untreated control plot has recorded pod damage of 10.82 per cent due to $H$. armigera.

\section{Grain yield}

In the management study maximum yield of 16.26 Q/ha. was obtained in treatment with NSKE at par with Bacillus thuringiensis kurstaki (Btk) (14.31 Q/ha.) followed by Beauveria bassiana (11.62 Q/ha.) and Karanj oil (10.42 Q/ha.) as against the lowest yield of (8.34 Q/ha.) in untreated control, during 2016 at S.K. CARS, Kawardha (Table 5 and Fig. 2).

During 2017-18, the maximum yield of 17.20 Q/ha. was obtained in treatment with NSKE at par with Bacillus thuringiensis kurstaki (Btk) $(15.52 \mathrm{Q} / \mathrm{ha}$.) followed by Beauveria bassiana (12.12 Q/ha.) and Karanj oil (11.46 $\mathrm{Q} / \mathrm{ha}$.) as against the lowest yield of 9.04 $\mathrm{Q} / \mathrm{ha}$. in untreated control.

On the basis of pooled mean data, the maximum yield of $16.73 \mathrm{Q} / \mathrm{ha}$. was obtained in treatment with NSKE at par with Bacillus thuringiensis kurstaki (Btk) (14.91 Q/ha.) followed by Beauveria bassiana (11.87Q/ha.) and Karanj oil (10.94 Q/ha.) as against the lowest yield of $8.69 \mathrm{Q} / \mathrm{ha}$. in untreated control. 


\section{References}

Ahmad, S., Ansari, M.S. and Muslim, M. 2015. Toxic effects of neem based insecticides on the fitness of Helicoverpa armigera (Hubner), Crop Protection. 68:72-78.

Anandhi, D. M. P., Elamathi, S. and Simon, S. 2011. Evaluation of biorational insecticides for management of Helicoverpa armigera in chick pea.Annals of Plant Protection Sciences. 19(1):207-209.

Anonymous, 2016. Kharif Progress report, Deputy Directorate of Agriculture. Government of Chhattisgarh, Kabirdham, Pp. 21-23.

Anonymous, 2017.Selected state wise area, production and productivity of gram in India. Ministry of agriculture and farmers welfare, Govt. of India.

Khatoon, N., Prakash J. 2004. Nutritional quality of micro wave cooked and pressure-cooked legumes. Int. J. Food
Sci. Nutr., 55(6):441-8.

Singh, S.K. and Yadav, D.K. 2009. Life table and biotic potential of Helicoverpa armigeraon chick pea. Ann. Pl. Protec. Sci., 17: 90-93.

Taggar, G.K. and Singh, R. 2012. Integrated management of Rabi Pulses In: Arora R. Singh B and Dhawan A.K. (ed). Theory and practices of integrated pest management PP 454-72.Scientific Publishers. India.

Wubneh, W. Yaregal., 2016. Biological control of chickpea pod borer, Helicoverpa armigera Hubner (Lepidoptera: Noctuidae): A global concern WSN 45(2): 92-110.

Zahra, A., Moosa, S., Samad, V., Vahid, M. and Ehsan, P. 2014. Acute, Sub lethal, and Combination Effects of Azadirachtin and Bacillus thuringiensis on the Cotton Bollworm, Helicoverpa armigera. Journal of Insect Science. 14:1-9.

\section{How to cite this article:}

Jai Kishan Bhagat, V. K. Soni and Chandrakar, H. K. 2020. Evaluation of Non-insecticidal Approaches against Pod Borer, Helicoverpa armigera Hubner on Chickpea at S.K. CARS, Kawardha Chhattisgarh. Int.J.Curr.Microbiol.App.Sci. 9(10): 218-226. doi: https://doi.org/10.20546/ijcmas.2020.910.028 